\title{
Value Based Healthcare: An Effective Leadership Tool to Build a Successful Healthcare Business Model
}

\section{Jazla Fadda*}

President and CEO, Visionary Mindset Professional and Management Development Training Institute, United Arab Emirates

*Corresponding Author: Jazla Fadda, President and CEO, Visionary Mindset Professional and Management Development Training Institute, United Arab Emirates.

Received: October 09, 2019; Published: October 11, 2019

DOI: $10.31080 /$ ASPS.2019.03.0416

\begin{abstract} healthcare business model. based health care to value-based health care as a business model for better successful healthcare facilities.

The review was held to answer the following questions

- What is the value based healthcare vs volume based healthcare

- How we measure the value in terms of outcomes and cost?

- What are the most effective tools to measure the outcomes of any health condition?

- What is the business model used to add value to healthcare?
\end{abstract}

Objective: (a)To review the main concept of value in healthcare, (b) to highlight on the relationship between quality and value of healthcare services (c) to use the three -tiers outcomes measures hierarchy as a tool for measuring value (d) to highlight on some leadership strategies for an effective value at different healthcare settings (e) to conclude a conceptual framework of a successful

Methodology: This paper uses a systematic review of some papers addressing the value based and quality based healthcare, besides reviewing five different case studies of some well -known healthcare entities, to generate the evidence of the role of shifting volume-

A search has been conducted to generate variety of sources, using computerized resources (Medline, Scopus and pro-quest). Findings were synthesized, summed up and interpreted to generate the business model. Different keywords were used to synthesis the inputs, such as: Value- based healthcare, healthcare quality, outcomes measures, healthcare business model, effective leadership in healthcare.

Keywords: Healthcare; Leadership; Business Model

\section{Background}

Improving performance of any kind of organization is relying on the shared goals and interests of all stakeholders. Value means the preeminent goal, it refers to the output achieved relative to the input (cost) incurred. We need to measure value to understand the performance of our organization as an essential approach for future improvements.
Value in healthcare has same yet unique concept. It's not a cost reduction, it should have imped the framework for performance improvement in health care. Institution of Medicine identifies 6 goals of healthcare delivery system: Safety, patient centeredness, efficiency, effectiveness, timeliness, and equity. Therefore, it should be going around the customer not the supplier, it's more related to the patient health outcomes attained per each dollar spent. Value 
is an essential element in healthcare industry, as it reflects what ultimately the customer (the patient) which can't be achieved unless unified all the stakeholder's efforts. Once the value improves, all stakeholders; patients, payers, insurer, providers and suppliers can also all benefit. Value implies different goals addressed in any healthcare settings, such as; quality, cost containment, profitability, safety, patient centered care, patient satisfaction, convenience, improving equity and accessibility.

\section{Key findings}

To generate an effective healthcare business model, we should consider how to sustain in the market by getting more financial stability. This sustainability can never be attained unless we provide value based healthcare. Value in healthcare should be measured by the outcomes, not the inputs, and not the outputs which is the volume of services delivered. "More care is not always better care, that's why we should shift our healthcare business model from the volume based services to value based ones. Measuring value is more related to the outcomes gained vs the cost needed, however, cost reduction without achieving the desired goals is very dangerous.

Value $=$ Outcomes $/$ cost. The nominator refers to the actual results attained in terms of patient health (patient survival, patient function status, sustainability, recovery). However, sometimes the results will also be affected by patient circumstances and preferences. That's why patient satisfaction is included as a main second part of the results or outcomes of any healthcare services provided. The dominator the cost, refers to the actual cost of the full cycle of care, in another say, including all the episodes of the services (interventions) provided for the patient's medical condition. Costs are related to services, devices, medications, equipment and ancillary inputs required to provide the service or intervention.

Value increases when better outcomes are attained at lower cost, outcomes improvement is a way to reduce costs, for instance early detection of disease will help using less invasive care and lead to faster recovery or less complications. Nevertheless, unnecessary use of screening is inefficient and will be value destructive. To reduce cost, we should spend money on high value services, such as preventive or early stage of care. Whereas, shifting the service to one provider to another or from one payer to another, won't add any value, but increasing administrative expenses.

Quality is the best approach to lower the cost, why? because quality is related to three aspects, structure, process and out- comes, it's related to the well-designed inputs require to deliver any kind of services, such as qualified professional and well-maintained equipment, technology, safe physical environment. Then the process that is safe for the patient and reducing medical errors, nosocomial infections or injuries. And finally, the outcomes which is better health status and patient satisfaction which will also be a measure tool to increase value and decrease the cost of repeating actions or miscommunication impeding results.

Measuring value can be conducted in a connected chain, starting from the preexisting conditions of the patient, then having appropriate structure of the facility and staff certification, following protocol and guidelines, then processing the services or interventions, and finally the outcomes generated. In between the process and outcomes, we should have clear measurable health indictors to evaluate the outcomes accordingly.

The outcomes for any medical condition can be measured using a three-tiered hierarchy. Tier 1 is the health status that is achieved, the first level, survival (mortality rate), the second level is the degree of health or recovery achieved or retained, such as freedom from disease and relevant aspects of functional status. Tier 2 outcomes are related to the recovery process, level 1 reflects the time of recovery, or best attainable function, level 2 reflects the disutility of the care or treatment process in terms of discomfort, retreatment, short-term complications, and errors and their consequences. Tier 3 is the sustainability of health. Level 1 related to recurrences of the original disease or longer-term complications, 2 nd level is related to the new health problems created because of treatment.

Three main strategies to increase value based healthcare were generated from this paper, boosting up the quality of care by improving the process for better outcomes and less wasted time and few health risks; setting a business plan based on value, as improving value is more important than short term fees for service profits, and care redesign for greater professional pride and a forwardlooking strategy. Value based strategies will lead to sustainability in the market by providing more financial stability, getting experience in managing risks by using evidence based care practices, positive relationships with physicians and healthcare stakeholders, building an advanced information system, and providing high-value based services in a responsible and sustainable means.

To improve VBHC, leaders should consider five main points for implementing outcomes measurements at their facilities. Find the 
believers, organize a cross-functional team with appropriate governance, invest time and resources, celebrate progress along the way, and use early successes to scale up and spread [1-8].

The concluded business model to pursue in healthcare is built up on four main pillars;

1. Healthcare utilization managed for efficiency and effectiveness through using data for re-designing data.

2. Disease management and care coordination through risk management and effective communication.

3. Workplace Guidelines that support achieving goals through workplace policies and collaborative efforts,

4. Focusing on Outcomes to provide quality and value through an effective leadership.

\section{Conclusion}

This article provides a systematic framework of a healthcare business model, using the value- based healthcare delivery concept. The business model was derived from the main concept of value in healthcare which is measured by the outcomes. The outcomes can be measured using a three-tiered hierarchy; health status that is achieved, the recovery process, and the sustainability of health. Attaining the value based healthcare required an effective leadership that find the believers, organize a cross-functional team, invest time and resources, celebrate progress along the way, and Use early successes to scale and spread. Moreover, to increase value based healthcare, three main strategies should be followed; boosting up the quality of care, setting a business plan based on value, care redesign for greater professional pride. following VBHC, will lead to sustainability and financial stability, better managing risks, creating advanced information systems. And finally building effective relationships with different healthcare stakeholders.

This paper could conclude and outline a framework for a successful healthcare business model by focusing on four main pillars; Healthcare utilization managed for efficiency and effectiveness, risk management and effective communication, workplace policies and collaborative efforts, and finally, outcomes to provide quality and value through an effective leadership.

\section{Bibliography}

1. Porter ME and Teisberg EO. Redefining health care: creating value-based competition on results. Boston: Harvard Business School Press (2006).
2. Institute of Medicine. Performance measurement: accelerating improvement. Washington, DC: National Academies Press (2006).

3. National Committee for Quality Assurance (NCQA). HEDIS and quality measurement: technical resources. NCQA.

4. Porter ME. "What is value in health care?" The New England Journal of Medicine 363 (2010): 2477-2481.

5. Jacob Lippa., et al. "What healthcare leaders should do to improve value for their patients". Harvard Business Review (2015).

6. Rebecca Weintraub., et al. "Measuring costs and outcomes in healthcare" (2015).

7. Laura S Kaiser and Thomas H Lee. "Turning value added based healthcare to a real business mode". (2015).

8. Siemens. Value based healthcare delivery: Building healthcare business model (2015).

\section{Volume 2 Issue 11 November 2019 (C) All rights are reserved by Jazla Fadda.}

